\title{
Development and characterization of a canine oral mucosa equivalent in a serum-free environment
}

\author{
Junhui Song, ${ }^{1}$ Kenji Izumi, ${ }^{1,2}$ Thomas Lanigan, ${ }^{3}$ Stephen E. Feinberg ${ }^{1}$ \\ ${ }^{1}$ Department of Oral and Maxillofacial Surgery, University of Michigan Medical Center, 1500 East Medical Center \\ Drive, Ann Arbor, Michigan 48109-0018 \\ ${ }^{2}$ Department of Oral and Maxillofacial Surgery, Niigata University, Niigata, Japan \\ ${ }^{3}$ Center of Gene Therapy, University of Michigan Medical Center, 1500 East Medical Center Drive, Ann Arbor, \\ Michigan 48109-0018
}

Received 2 April 2004; revised 3 June 2004; accepted 15 June 2004

Published online 18 August 2004 in Wiley InterScience (www.interscience.wiley.com). DOI: 10.1002/jbm.a.30144

\begin{abstract}
The objectives of this study were to develop a serum-free system for culturing canine oral keratinocytes, the construction and characterization of a canine ex vivo produced oral mucosa equivalent (EVPOME), and transduction green fluorescent protein (GFP) into keratinocytes as a post-grafting tracking marker. Dissociated canine buccal mucosa keratinocytes were cultured in a chemically defined serum-free medium, Epilife ${ }^{\mathrm{TM}}$. First-passage keratinocytes were transfected with the GFP gene using a lentiviral vector, sorted by flow cytometer and seeded onto a dermal equivalent, AlloDerm ${ }^{\circledR}$ to form EVPOMEs. The EVPOME was characterized by histology and immunohistochemistry, for p63, Ki-67, and involucrin. Laser confocal microscopy was used to locate GFP-transfected keratinocytes within the EVPOME. Cultured canine oral keratinocytes grew rapidly over the first three passages and then the proliferative rate
\end{abstract}

decreased. The canine EVPOME formed a well-stratified epithelial layer. The majority of p63 and Ki-67 immunopositive cells were located in the basal layer whereas cytoplasmic involucrin expression was seen in the suprabasal layers, similar to native canine buccal mucosa. Under laser confocal microscopy, significant green fluorescence was observed throughout the EVPOME. In conclusion, canine EVPOMEs were successfully fabricated in a defined serum-free system with similar characteristics to native buccal mucosa. GFPtransfected canine oral keratinocytes could be identified within the EVPOME. (C) 2004 Wiley Periodicals, Inc. J Biomed Mater Res 71A: 143-153, 2004

Key words: canine model; keratinocyte; serum-free culture system; ex vivo produced oral mucosa equivalent (EVPOME); green fluorescent protein (GFP)

\section{INTRODUCTION}

Oral and maxillofacial surgeons are frequently confronted with a shortage of oral mucosa for reconstruction of the oral cavity. Routine use of split-thickness skin grafts has disadvantages, such as presence of adnexal structures and a different pattern of keratinization. Cultured autologous oral keratinocyte sheets and oral mucosa equivalents constructed on collagen dermal equivalents are easily degraded and not suited for intraoral clinical applications. ${ }^{1,2}$ We have previously developed a human ex vivo produced oral mucosa equivalent (EVPOME) by culturing human oral keratinocytes and seeding them onto a cadaveric der-

Correspondence to: S. E. Feinberg; e-mail: sefein@umich.edu Contract grant sponsor: Kato Memorial Bioscience Foundation (to KI)

Contract grant sponsor: US PHS; contract grant number: NIDCR/NIH R01 DE 13417 (to SEF)

(C) 2004 Wiley Periodicals, Inc. mal equivalent, AlloDerm ${ }^{\circledR}$ (Lifecell Corp., Branchburg, NJ), in a serum-free culture system without using an irradiated 3T3 xenogeneic feeder cell layer. The seeded human oral keratinocytes formed a stratified epithelial layer on the dermal component of the EVPOME when it was raised to an air-liquid interface, developing into an epithelial layer that was similar to the original oral mucosa used to fabricate it. ${ }^{3}$ Subcutaneous grafting of human EVPOME into SCID mice showed the importance of the presence of a mature epithelia in the growth and formation of microvessels within the dermis of the EVPOME as compared with the dermal equivalent, AlloDerm ${ }^{\circledR}$, lacking an epithelial layer. ${ }^{4}$ Human EVPOMEs used clinically in patients to reconstruct oral defects showed the persistence of epithelial cells on the grafts at 7 days after transplantation by cytological smear. In addition, postoperatively, the epithelial layer of the grafted EVPOME appeared better organized, and the inflammatory response in underlying the dermal equivalent was less pronounced than the AlloDerm ${ }^{\circledR}$ grafts lack- 
ing an epithelial layer, demonstrating the importance of the barrier effect of the grafted epithelial layer. ${ }^{2}$ It is important to assess the longevity and ultimate fate of the grafted epithelium on the EVPOMEs. To be able to do this appropriately will necessitate labeling transplanted oral keratinocytes to be able to differentiate them from ungrafted cells. These are studies that cannot ethically be performed on humans, thus the need for a comparable animal model, such as the dog, that would allow these and other in vivo studies to be performed.

Our research team has successfully fabricated a human tissue-engineered oral mucosa in a defined medium free of serum and a xenogeneic feeder layer that will be used in an upcoming clinical trial. ${ }^{5}$ The dog presents an excellent animal model for performing identical intraoral studies as are planned in our clinical trial because it has similar soft tissues and is large enough to do surgical procedures that are performed in humans. ${ }^{6-8}$ To be able to extrapolate the studies performed in dogs to our human EVPOME, the canine EVPOME needs to be fabricated in a manner that is consistent with the process of fabricating human EVOPME, following Food and Drug Administration (FDA) guidelines for cells grown in vitro that are grafted back in humans. ${ }^{5}$ To date, several investigations that have attempted to culture canine oral keratinocytes used irradiated 3T3 xenogeneic fibroblast as a feeder layer, and/or a high concentration of serum in the medium. ${ }^{9-13}$ The use of a 3T3 feeder layer and/or serum places the cultured oral keratinocytes at a risk of cross-contamination with viral and/or other material, such as prions and conflicts with FDA guidelines. Crameri et al. ${ }^{9}$ indicated that several commercial serum-free culture media, such as KGM ${ }^{\mathrm{TM}}$ (Cambrex, Santa Rosa, CA) and KSFM ${ }^{\mathrm{TM}}$ (Gibco, Grand Island, $\mathrm{NY}$ ), are only effective on normal human oral keratinocytes, and neoplastic canine oral keratinocytes but not on normal canine oral keratinocytes. Ophof et al. ${ }^{11}$ have compared various materials to construct a canine oral mucosa equivalent, including AlloDerm ${ }^{\circledR}$, and several variations made from type 1 collagen. The seeded canine oral keratinocytes only formed a continuous epithelial layer, similar to native mucosa on the AlloDerm ${ }^{\circledR}$ dermal equivalent. It demonstrated that a synthetic dermal substitute, such as collagen, could only be effective in forming a basement membrane and epithelial layer when fibroblasts were present within the dermal equivalent, otherwise the seeded keratinocytes would migrate into the underlying porous collagen and form cell clusters or cysts. It was evident that we needed to develop a technique to fabricate a canine EVPOME, similar to human EVPOME, in a defined serum-free system devoid of a feeder layer. The results from the canine in vivo investigations could then be extrapolated to humans and used for eventual FDA approval for human EVPOME.
Thus, the objectives of this study were to:

1. develop a serum-free system without a feeder layer for predictable culturing of canine oral keratinocytes,

2. construct a full-thickness oral mucosa equivalent, in vitro, in a chemically defined system,

3. characterize the developing epithelium on the canine EVPOME, and

4. transduce a reporter gene, green fluorescent protein (GFP), into canine oral keratinocytes, with a lentivirus vector, to serve as a marker for grafted cells.

\section{METHODS}

\section{Cell isolation and culture}

The oral cavity of five euthanized mongrel dogs was prepped with Betadine (Purdue Frederick, Stamford, CT), and samples of buccal oral mucosa tissue were obtained within $30 \mathrm{~min}$ after death. The excised tissues were washed twice in Dulbecco's phosphate-buffered saline (PBS) (BD Whittaker, Walkersville, MD) supplemented with 125 $\mu \mathrm{g} / \mathrm{mL}$ Gentamycin and $1 \mu \mathrm{g} / \mathrm{mL}$ Fungizone (Gibco), and kept in the second washing for $30 \mathrm{~min}$ to remove any residual bacteria. After the subcutaneous tissue was trimmed off, samples were cut into 2- to 3-mm-wide strips, digested in $0.1 \%$ trypsin (Sigma, St. Louis, MO) at room temperature overnight (16-18 h). The same concentration of trypsin inhibitor (Sigma) was then used to neutralize the trypsin. The epidermal side of the mucosa was gently scraped with a scalpel to detach the keratinocytes. The resultant cell suspension was filtered through a 400-micron mesh, centrifuged at $1000 \mathrm{rpm}$ for $5 \mathrm{~min}$, resuspended in Epilife ${ }^{\mathrm{TM}}$ keratinocyte serum-free medium (Cascade Biologics, Portland, OR) supplemented with $0.06 \mathrm{mM}$ calcium, $25 \mu \mathrm{g} / \mathrm{mL}$ Gentamycin, and $0.375 \mu \mathrm{g} / \mathrm{mL}$ Fungizone, and then seeded into flasks at an initial density of $2 \times 10^{5}$ cells $/ \mathrm{cm}^{2}$. Cells were incubated at $37^{\circ} \mathrm{C}, 5 \% \mathrm{CO}_{2}$ in humidified air; medium was changed every other day. Once the keratinocyte cultures attained $70-80 \%$ confluence, they were split and passaged by washing with PBS, digested with $0.025 \%$ trypsin/ethylenediaminetetraacetic acid (Cascade Biologics), neutralized by defined $0.0125 \%$ trypsin inhibitor (Cascade Biologics), centrifuged, and resuspended at a seeding density of $2 \times 10^{4}$ cells $/ \mathrm{cm}^{2}$. Keratinocytes were then placed into serial culture and carried until their proliferative ability was exhausted. The seeding cell density of both the canine oral keratinocyte primary culture and subcultures was similar to the cell density used in our human keratinocyte cultures. ${ }^{3}$

\section{Growth curve}

To create a growth curve, the primary harvested keratinocytes were seeded into a 24-well plate (Corning Inc., Corn- 
ing, NY) at an initial density of $2 \times 10^{5}$ cells/well. To monitor the proliferation rate of cells, two wells were trypsinized and counted every day up to 12 days after seeding. Cell counts of each well were duplicated; mean value was taken as the result. Growth curves were plotted as cell number on a log scale, against time on a linear scale. Data were presented as mean $\pm \mathrm{SD}$. The lag time, population doubling time, and plateau density of primary cells were then determined as described elsewhere. ${ }^{14}$ Briefly, the cumulative total cell number of cells was determined at each passage based on the assumption that all cells from the previous passage had been replated. ${ }^{3}$ The population doubling time of passage one and passage two cells was calculated from the formula $\mathrm{PD}=\left[\log \left(N / N_{0}\right)\right]^{-1}$, where $N=$ cell number at harvest, $N_{0}=$ cell number inoculated, PDT $=$ $\mathrm{I} / \mathrm{PD}$, and I = interval (days) between the passages. ${ }^{15}$

\section{Forward scatter count by flow cytometry}

To further evaluate cell size and sort for labeled canine keratinocytes grown in the Epilife ${ }^{\mathrm{TM}}$ serum-free culture system, cells from each passage were trypsinized, pelleted, and fixed in ice cold $90 \%$ methanol to a final density of $1 \times$ $10^{6} / \mathrm{mL}$, then stored at $-20^{\circ} \mathrm{C}$ until use for flow cytometric analysis. Cells were spun down and resuspended in an equal amount of PBS before flow cytometry. Relative cell size was determined by measuring forward and orthogonal light scatter in a Coulter Flow Cytometer (Beckman Coulter ESP, USA).

\section{GFP gene transduction and flow cytometry}

Dog oral keratinocytes from passage one or two were seeded into a six-well plate. On the second day, $1.25 \mathrm{~mL}$ of $10 \times$ concentration of FIV34TF10/huGFP lentivirus resuspended in Epilife ${ }^{\mathrm{TM}}$ media (Vector Core; University of Michigan, Ann Arbor, MI) was mixed with $8 \mu \mathrm{g} / \mathrm{mL}$ polybrene and added to the cells. The plate was then spin inoculated for $90 \mathrm{~min}$ at $2500 \mathrm{rpm}$ (Eppendorf Centrifuge 5810) and then incubated at $32^{\circ} \mathrm{C} / 5 \% \mathrm{CO}_{2}$. After $22 \mathrm{~h}$, the medium was changed, and then subsequently changed every $48 \mathrm{~h}$. One week later, keratinocytes were harvested and resuspended in PBS at $1 \times 10^{6} / \mathrm{mL}$ with propidium iodide $400 \mu \mathrm{L} / \mathrm{mL}$ (Sigma, St. Louis, MO), then sorted by flow cytometry (FACS Vantage SE; Becton Dickinson, San Jose, CA) according to GFP expression. The positive cells were harvested in culture medium and seeded onto the dermal equivalent, AlloDerm ${ }^{\circledR}$. As a negative control, we seeded nontransduced cells from the same dog oral keratinocyte population.

\section{Fabrication of canine EVPOME}

Circular pieces of AlloDerm ${ }^{\circledR}, 1.0 \mathrm{~cm}$ in diameter, were rehydrated and coated with $5 \mu \mathrm{g} / \mathrm{cm}^{2}$ type IV collagen (Fluka, Buchs, Swizerland) as described previously. ${ }^{3}$ Kera- tinocytes from passage one or two, which is the passage number that is planned to be used in our human clinical trial, were resuspended in high calcium $\left(1.2 \mathrm{mM} \mathrm{Ca}^{++}\right)$ Epilife $^{\mathrm{TM}}$ medium and seeded onto the AlloDerm ${ }^{\circledR}$ at the density of $2.5 \times 10^{5}$ cells $/ \mathrm{cm}^{2}$. Medium was changed every day. On day 4 , the AlloDerm ${ }^{\circledR} /$ oral keratinocyte composites were transferred to an air-liquid interface. Medium was changed every other day. To assess the histological character of the canine EVPOME, EVPOMEs were fixed with $10 \%$ formalin at day 4,11 , and 18, after initial seeding of oral keratinocytes, processed for routine histology, and $5-\mu \mathrm{m}-$ thick sections were stained with hematoxylin and eosin. For GFP-transfected EVPOME, frozen sections of 7- $\mu \mathrm{m}$ thickness were examined by a laser confocal microscope (MCR600; Bio-Rad, UK) using a wavelength of $488 \mathrm{~nm}$.

\section{Immunohistochemistry}

Immnohistochemistry analysis was performed on native canine oral mucosa tissue on day 4, 11, and $18 \mathrm{EV}$ POMEs to detect a stem cell marker, p63 (clone 4A4; Pharmingen, San Diego, CA), a cell proliferation marker, Ki-67 (clone Ki-67; Beckman Coulter, Fullerton, CA), and a cell differentiation marker, involucrin (ab68; Abcam, Cambridge, UK). For staining of p63 and Ki-67, samples were fixed with $10 \%$ formalin, embedded in paraffin, and cut at $5 \mu \mathrm{m}$. After deparaffinizing, quenching of endoperoxidase activity was performed by 30 -min incubation in $0.3 \% \mathrm{H}_{2} \mathrm{O}_{2}$ with methanol. Antigen retrieval technique was used for immunohistochemistry of p63 and Ki-67 by microwave heating for $20 \mathrm{~min}$ in citrate buffer, $\mathrm{pH}$ 6.0. Sections were incubated with mouse monoclonal antihuman antibody to p63 (1:150), Ki-67 (1:80) overnight at $4{ }^{\circ} \mathrm{C}$. For involucrin, a $7-\mu \mathrm{m}$ frozen section was used and incubated with mouse monoclonal anti-human involucrin antibody (1:250) for $2 \mathrm{~h}$ at room temperature. Antibody binding was performed with an anti-mouse immunoglobulin $\mathrm{G}$ biotinylated antibody and avidin biotin complex solution according to the protocol recommended by the manufacturer and visualized by DAB (3' $3^{\prime}-$ diaminobenzidine) technique (Vector Laboratories, Burlingame, CA). Sections were counterstained with hematoxylin. The cell counting method of immunopositive cells was the same as previously stated. ${ }^{3}$

\section{RESULTS}

\section{Cell isolation and culture}

Phase-contrast microscopy

Under phase-contrast microscopy, cultured primary keratinocytes attached to the bottom of the flasks and had a spindly cell shape and ruffled membrane. It appeared that numerous keratinocytes migrated by extending long "foot-like" appendages 

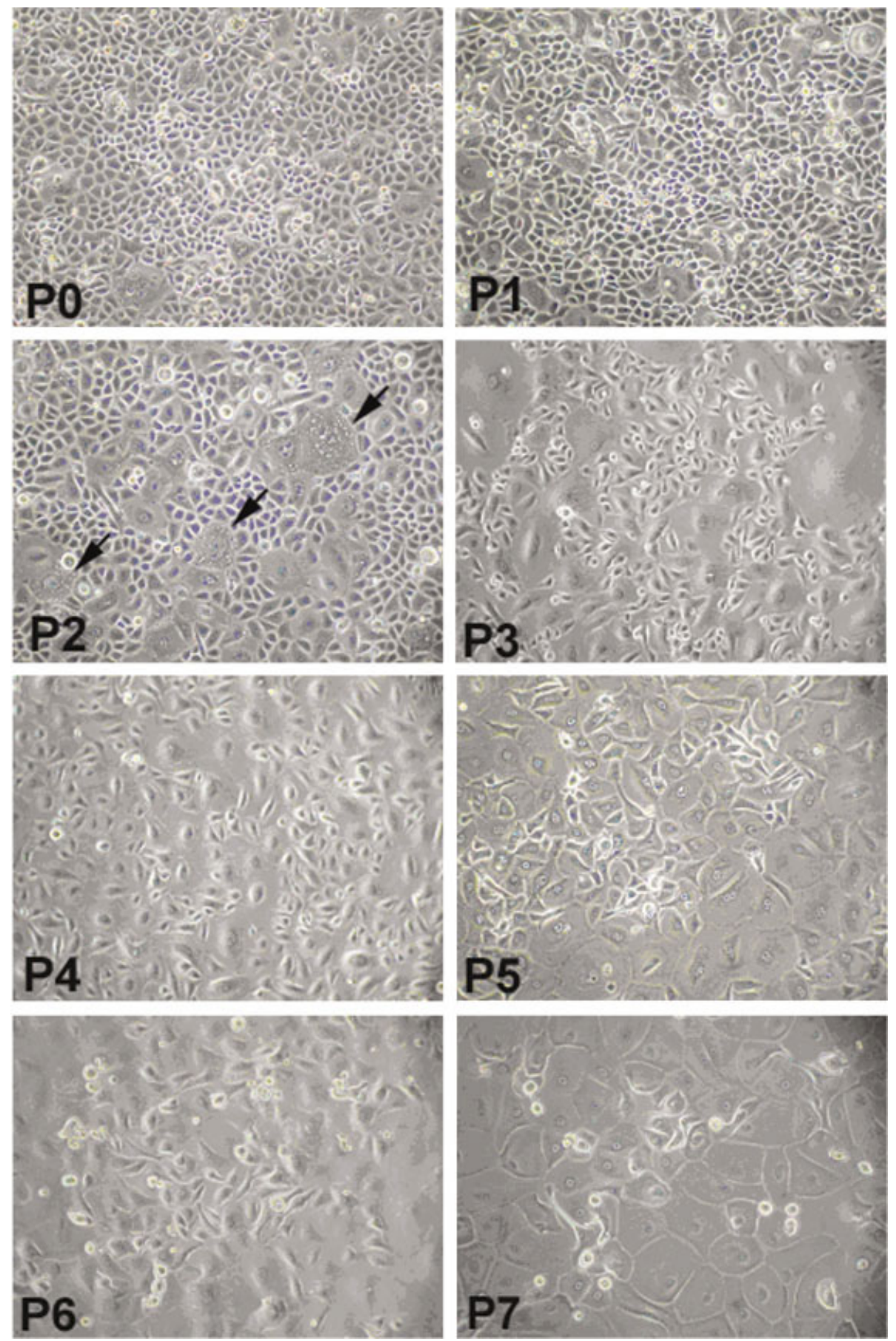

Figure 1. Phase-contrast micrographs of dog oral keratinocytes from generation passage P0 to P7 (original magnification, $\times 100$ ). Arrows in passage two cells (P2) indicate larger keratinocytes containing intracytoplasmic granules. [Color figure can be viewed in the online issue, which is available at www.interscience.wiley.com.]

(pseudopodia). On confluence, the interface between the keratinocytes became narrower with cells gradually assuming a polygonal shape with a pavement-like appearance. In our culture system, canine oral keratinocytes could be passaged for, at the most, seven generations. In primary culture, the majority of the cells had a small polygonal shape with several larger irregular keratinocytes scattered among them. With each passage, the proportion of larger cells increased. From passage two on, the cells appeared as large "differentiated" keratinocytes containing numerous granules within their cytoplasm (Fig. 1). 


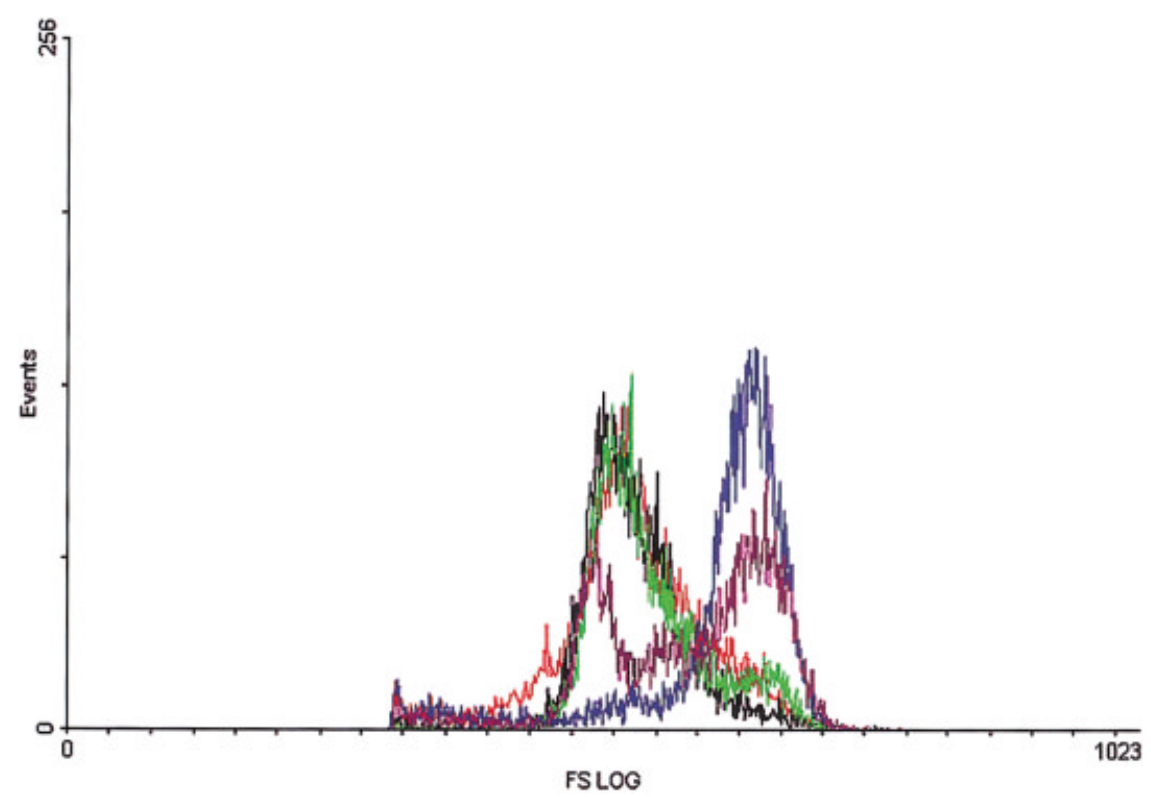

Figure 2. Flow cytometric analysis of relative cell size of canine oral keratinocytes from passage P0 to P5 (each of the five colors represents a different generation: red $=\mathrm{P} 1$, black $=\mathrm{P} 2$, green $=\mathrm{P}$, blue $=\mathrm{P} 4$, purple $=$ P5). [Color figure can be viewed in the online issue, which is available at www.interscience.wiley.com.]

Flow cytometry analysis

The change of cell size on passage was confirmed by flow cytometry analysis of relative cell size. The average cell size increased from passage one to passage five (no analysis for passages six and seven was done because of the limited cell numbers available). The peak of the forward scatter histogram shifted to the right along the $x$ axis from passage one to passage four. In passage five, there were two peaks showing the disparity between large and small keratinocytes (Fig. 2).

\section{Cell growth kinetics}

The keratinocyte expansion of each generation increased continuously from primary cell to passage three. However, after passage three, the cell output decreased sharply (Fig. 3) as opposed to human oral keratinocytes which were able to be passaged up to 10 times (ref. 3 and unpublished data). The total expansion ratio varied among the five different dogs from 1:2.5 to 1:8.8 PDT of P1 was 2.29 days; PDT of P2 was 2.96 days. From the data of five dogs, the average lag time of primary keratinocytes was 2 days. From day 3 to day 8 , the curve significantly correlated to the $\log$ model. When the cells became confluent at day 8 , the saturation density was $1.57 \times 10^{5}$ cells $/ \mathrm{cm}^{2}$ (Fig. 4).

\section{In vitro culture of canine oral mucosa equivalent}

\section{Histology}

After culturing canine composites submerged for 4 days, a keratinocyte monolayer was seen attached to the AlloDerm ${ }^{\circledR}$. On day 11, after culturing at an airliquid interface for 7 days, the monolayer of oral keratinocytes stratified to more than three cell layers thick. The basal cell nuclei were cubic, whereas the suprabasal cell nuclei were oval with the long axis parallel to the surface of the AlloDerm ${ }^{\circledR}$. The superficial two to three cell layers were eosinophilic, showing parakeratinization, with a narrow nucleus seen within

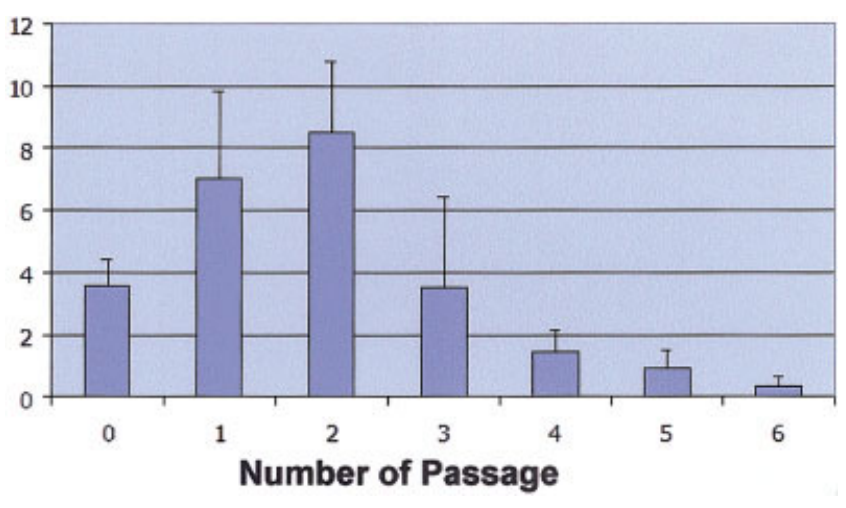

Figure 3. The harvested cell number of each passage of canine oral keratinocytes (representative of five samples); $y$ axis $=$ number of total cell output $\left(\times 10^{6}\right)$. 


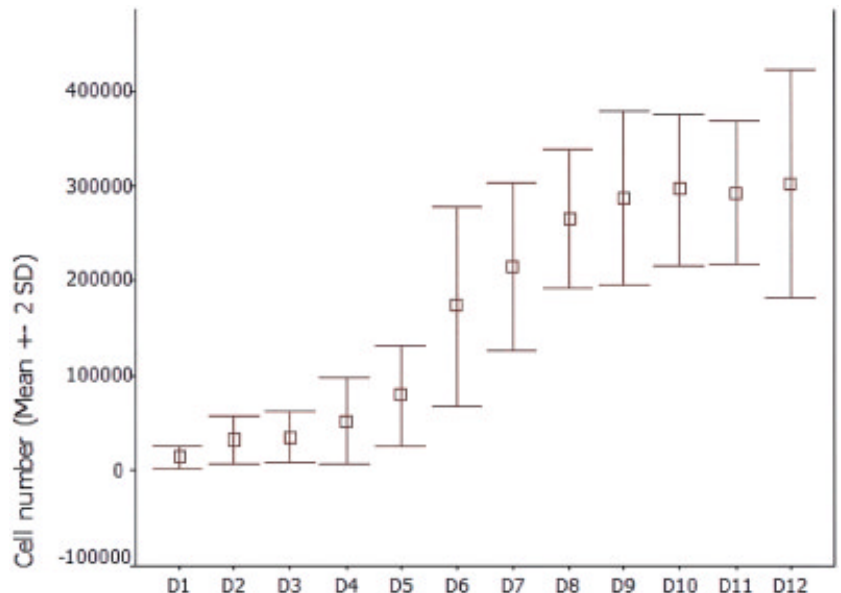

Figure 4. Growth curve of primary canine oral keratinocyte (representative of five samples).

the cells. On day 18, cultured at an air-liquid interface for 14 days, three different cell layers were observed: the basal cell layer was composed of compacted columnar cells, the suprabasal cell layer of oval or irregular-shaped keratinocytes with an eccentrically placed nucleus, and the superficial cell layer, which contained more than 20 parallel cell layers, with flat nuclei. The 18-day EVPOME was similar to native canine oral mucosa, lacking only the deep rete pegs ridges and pigmentation (Fig. 5).

\section{Immunohistochemistry}

In native canine oral buccal mucosa, p63 nuclear expression was seen in the basal and suprabasal layers, at the base of the epidermal bulge into the dermis. On day 4 equivalents, a majority of the cells within the monolayer showed positive nuclear immunoreaction with p63; on day 11 equivalents, the p63 immunopositive cells were located mainly in the basal and the lowest suprabasal cell layers; and on day 18 equivalents, positive cells were found in both the basal and suprabasal cell layers. The percentage of positively stained p63 cells was higher than seen in native canine tissue (Fig. 6). Nuclear Ki-67 expression, overall, was less than seen with p63. Day 4 equivalents showed few positive cells; in day 11 and day 18 equivalents, positive cells were only observed in the basal cell layer, this was in comparison to only several positive cells noted in native buccal mucosa (Fig. 7). Involucrin expression was found in the cytoplasm of the suprabasal cell layer in native oral buccal mucosa. In day 4 equivalents, there was no expression, whereas in day 11 and day 18 equivalents, immunoreaction was seen in the superior portions of the suprabasal cell layers (Fig. 8; Table I).

\section{GFP transfection and confocal microscopy}

The average transduction efficiency of canine oral keratinocyte was $26 \%$ (Fig. 9). There was no morphologic change of the keratinocytes after transduction as seen under a phase-contrast microscope. After sorting
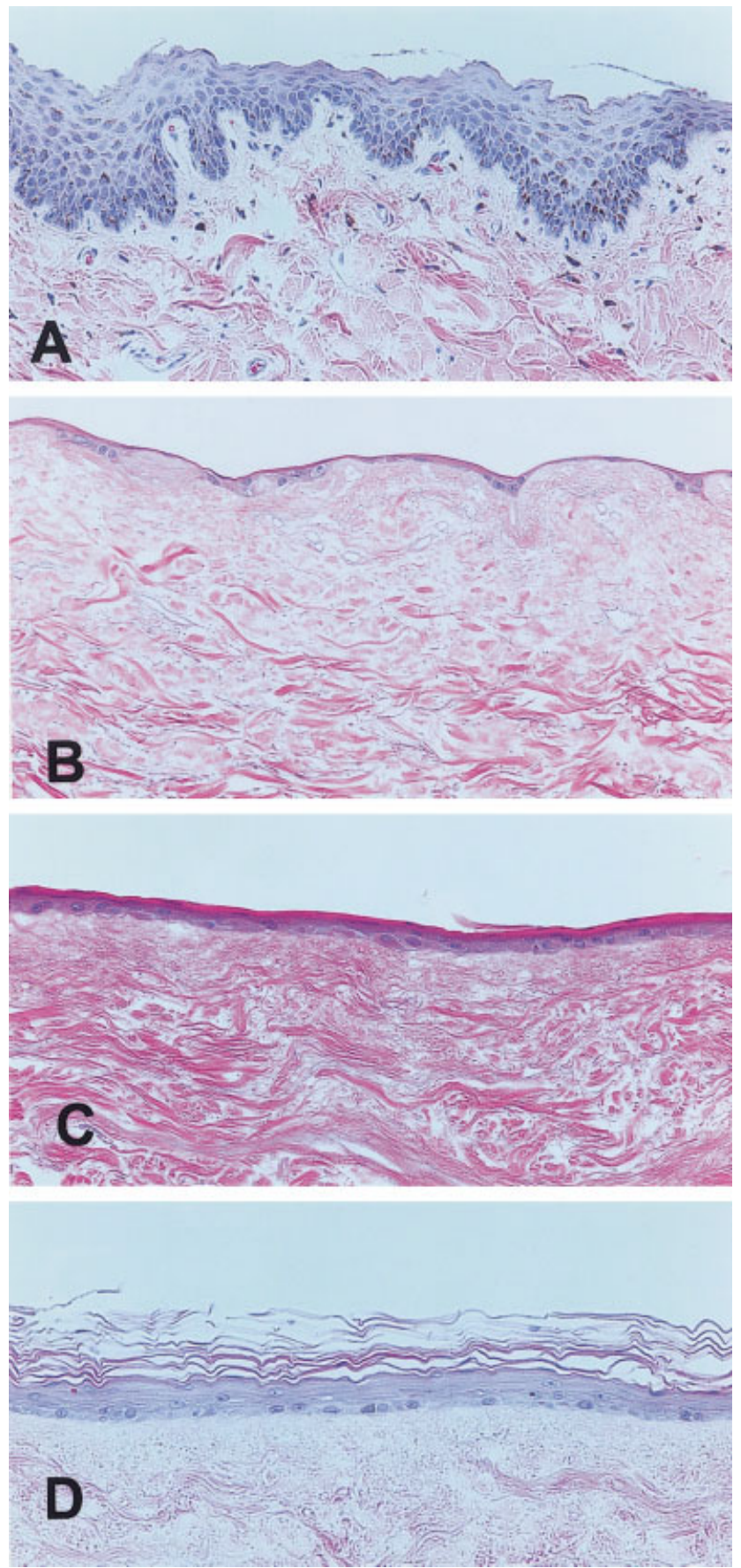

Figure 5. Histological findings of native canine oral mucosa (A) and canine ex vivo produced oral mucosa equivalent of day 4 (B), day 11 (C), and day 18 (D) (hematoxylin and eosin; original magnification, $\times 200$ ). [Color figure can be viewed in the online issue, which is available at www. interscience.wiley.com.] 


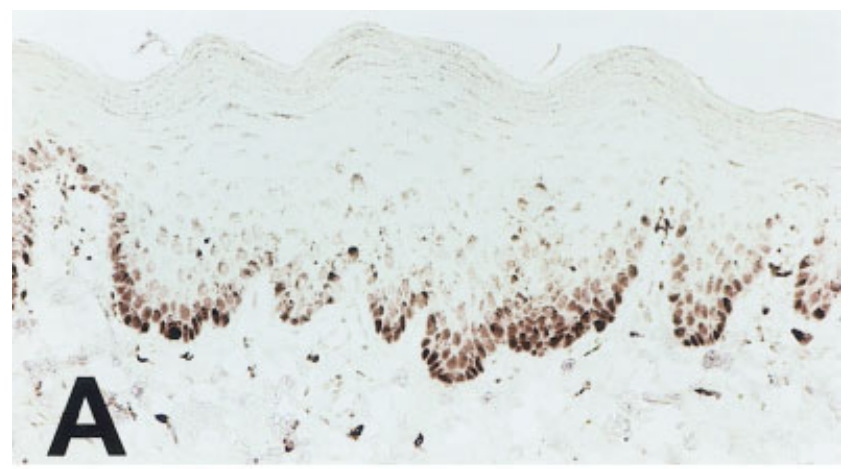

\section{DISCUSSION}

A chemically defined culture system, free of serum, a xenogeneic feeder layer, and pituitary extract allows a more accurate control of culture conditions in vitro, reduced variability in qualitative and quantitative culture medium composition, elimination of potential sources of (micro-) biological contamination, and the advantage of down-stream processing (isolation of cell culture products). ${ }^{16}$ In addition, reagents such as fetal
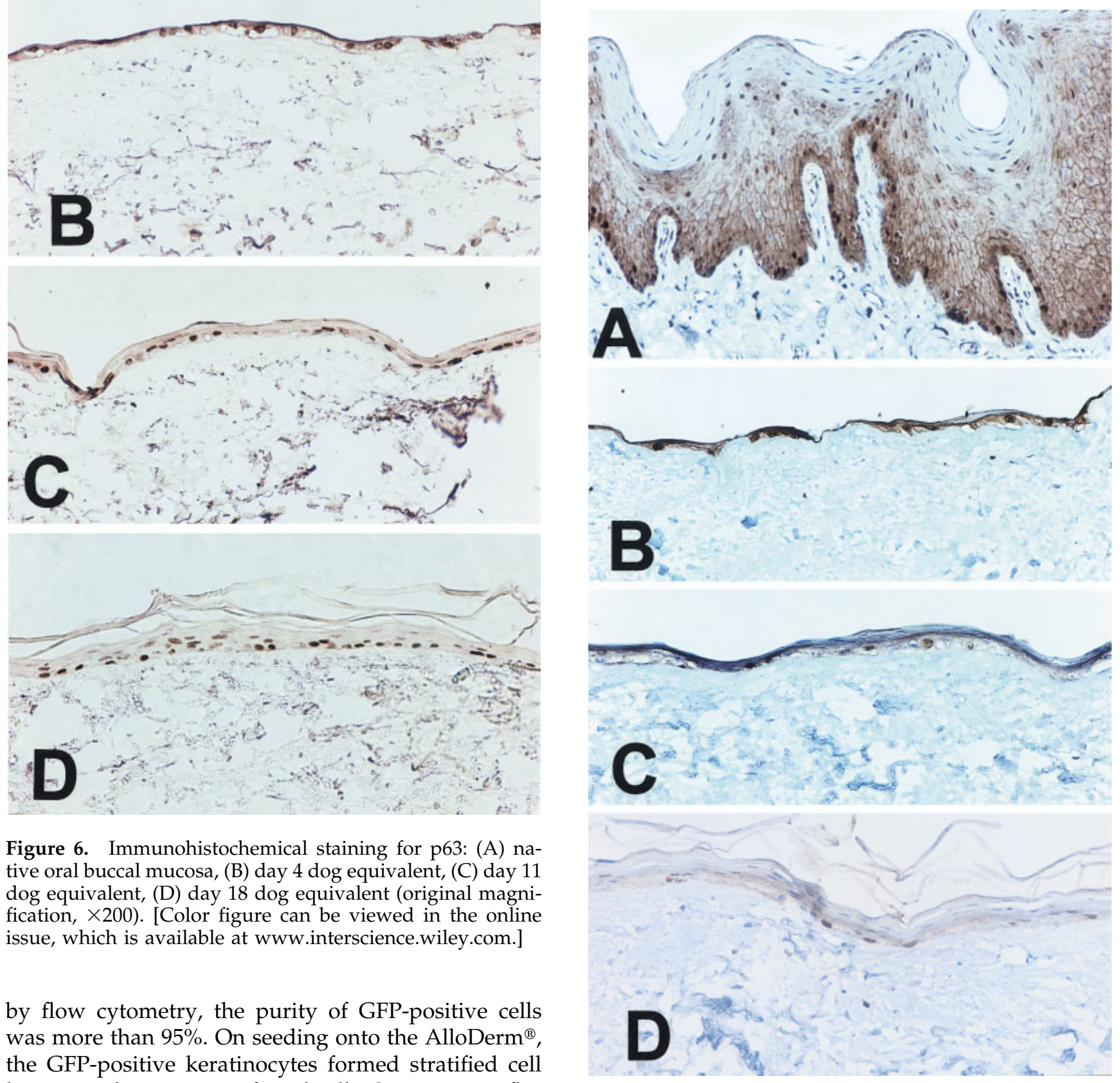

Figure 6. Immunohistochemical staining for p63: (A) native oral buccal mucosa, (B) day 4 dog equivalent, (C) day 11 dog equivalent, (D) day 18 dog equivalent (original magnification, $\times 200$ ). [Color figure can be viewed in the online issue, which is available at www.interscience.wiley.com.]

by flow cytometry, the purity of GFP-positive cells was more than $95 \%$. On seeding onto the AlloDerm ${ }^{\circledR}$, the GFP-positive keratinocytes formed stratified cell layers similar to untransfected cells. Strong green fluorescence was observed on day 11 equivalents using confocal microscopy when compared with the negative control containing nontransfected keratinocytes (Fig. 10).

Figure 7. Immunohistochemical staining for Ki-67: (A) native oral buccal mucosa, (B) day 4 dog equivalent, (C) day 11 dog equivalent, (D) day 18 dog equivalent (original magnification, $\times 200$ ). [Color figure can be viewed in the online issue, which is available at www.interscience.wiley.com.] 


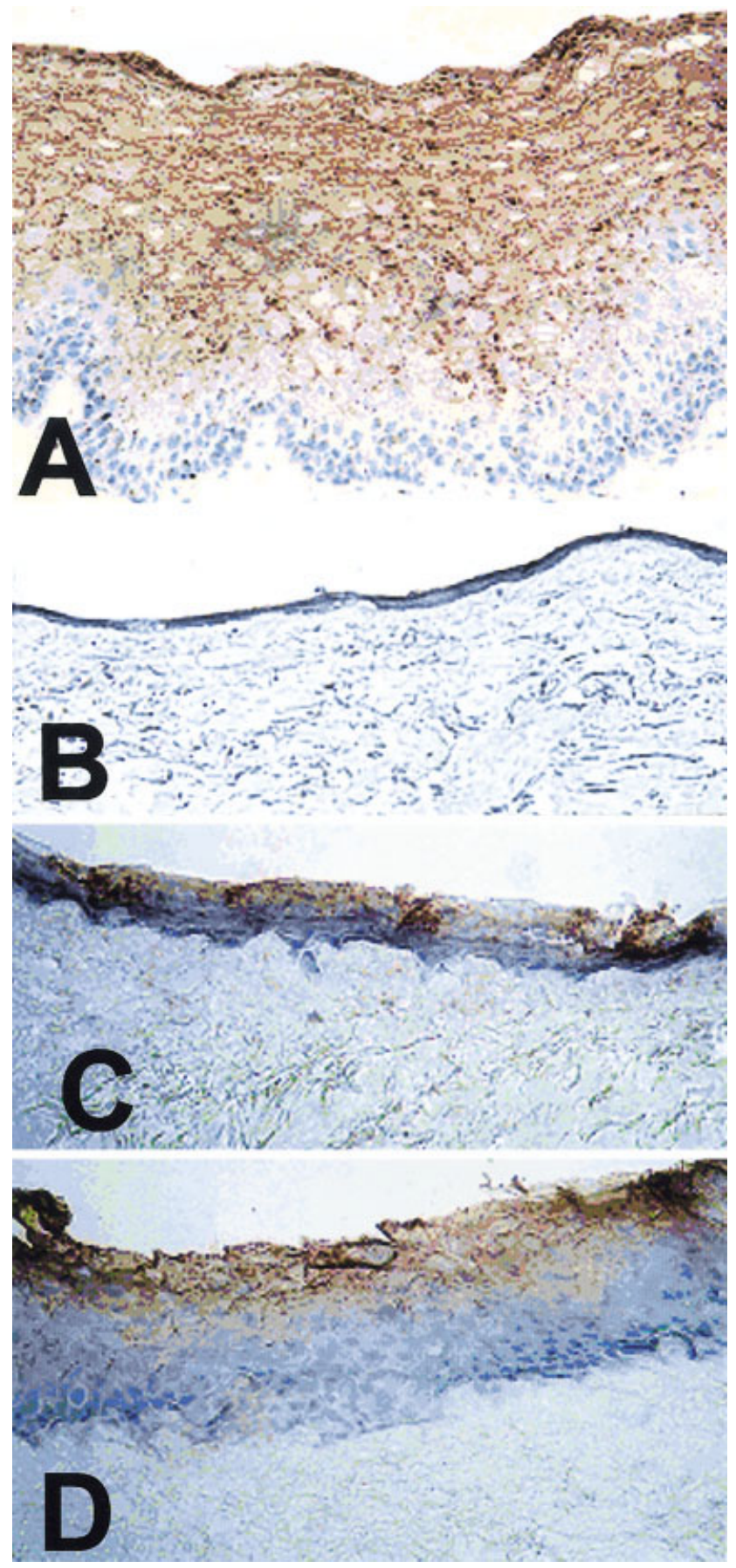

Figure 8. Immunohistochemical staining for involucrin: (A) native oral buccal mucosa, (B) day 4 dog equivalent, (C) day 11 dog equivalent, (D) day 18 dog equivalent (original magnification, $\times 200$ ). [Color figure can be viewed in the online issue, which is available at www.interscience.wiley. com.]

calf serum used in cell culture medium is difficult to remove during washing and can trigger an immunologic reaction in patients who receive cell infusions, thus negating its use if human clinical trials are being considered. ${ }^{17}$ The development of a serum-free culture medium for cells and, in particular, human kera-
TABLE I

Comparison of Percentage of Immunopositive Cells Between Native Oral Buccal Mucosa and Day 18 Equivalent

\begin{tabular}{lccccc}
\hline & \multicolumn{2}{c}{$\begin{array}{c}\text { Native Buccal Oral } \\
\text { Mucosa }\end{array}$} & & Day 18 & Equivalent \\
\cline { 2 - 3 } \cline { 6 - 7 } Antibody & Basal & Suprabasal & & Basal & Suprabasal \\
\hline p63 & +++ & + & & ++ & ++ \\
Ki-67 & ++ & + & & ++ & - \\
Involucrin & - & +++ & & - & ++ \\
\hline
\end{tabular}

Proportion of positive cells:,$+++>50 \% ;++,>10 \%$ and $<50 \%$;,$<10 \%$; and,$- 0 \%$.

tinocytes, has allowed marked advances to be made in the area of keratinocyte biology. ${ }^{18}$

With this in mind, it is unfortunate, to date, that there is no serum-free medium available to culture animal (canine or porcine) keratinocytes. Crameri et al. ${ }^{9}$ found that two commercial serum-free media, $\mathrm{KGM}^{\mathrm{TM}}$ and $\mathrm{KSFM}^{\mathrm{TM}}$, which performed well in human keratinocyte cultures, failed to support normal canine oral keratinocyte growth consistently after the first passage. The $\mathrm{KGM}^{\mathrm{TM}}$ and $\mathrm{KSFM}^{\mathrm{TM}}$ media that were used contained a higher concentration of calcium, 0.15 $\mathrm{mM}$ and $0.09 \mathrm{mM}$, respectively, when compared with $0.06 \mathrm{mM}$ seen in Epilife ${ }^{\mathrm{TM}}$ used in our investigation. Studies have shown that keratinocytes grown in a calcium concentration less than $0.07 \mathrm{mM}$ proliferate more rapidly and tend to differentiate more slowly, stratify less, form less intercellular contacts, and form fewer cornified envelopes. ${ }^{19}$ When the calcium concentration increases to higher levels, keratinocytes begin to differentiate and express the appropriate markers. ${ }^{19}$ This might be the main reason why we have been able to continuously culture our canine oral keratinocytes up to seven passages in our experiments. In

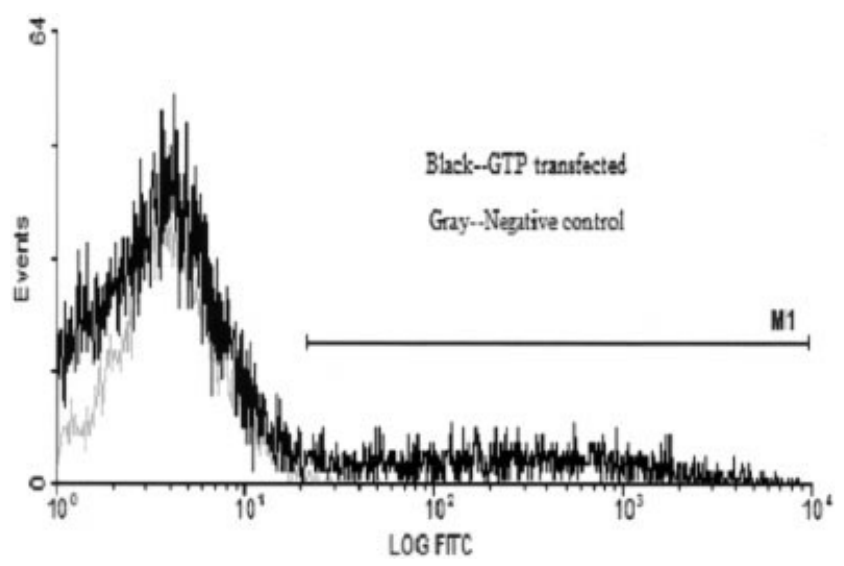

Figure 9. Flow cytometry analysis of GFP-positive cells after transduction of canine oral keratinocytes. Arrow (M1 cursor) indicates the borderline (range) showing positive fluorescein isothiocyanate (FITC) fluorescence when compared with negative control. 

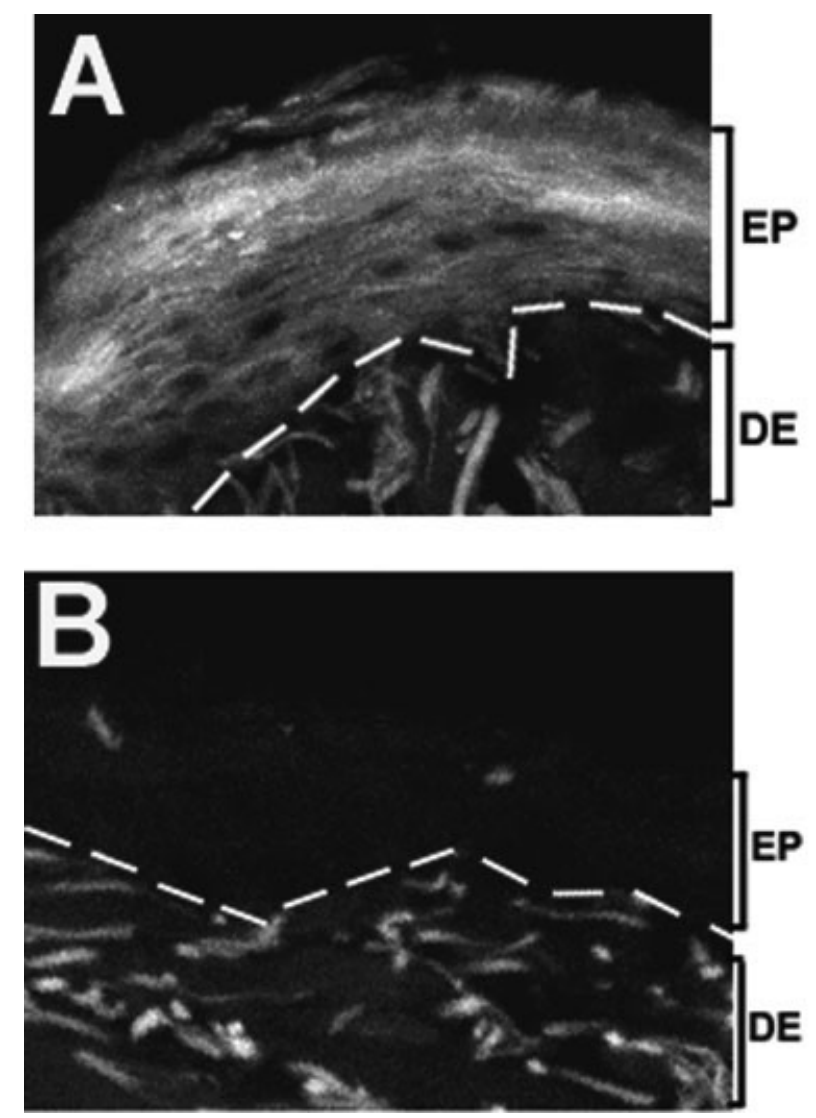

Figure 10. Laser confocal microscopy: (A) strong green fluorescence in EVPOMEs fabricated with GFP-transduced dog oral keratinocytes, (B) minimal green autofluorescence in EVPOMEs fabricated with nontransduced dog oral keratinocytes (original magnification, $\times 100$ ). White broken line shows an interface between epithelial layer (EP) and dermal component, AlloDerm ${ }^{\circledR}$ (DE).

addition, both $\mathrm{KGM}^{\mathrm{TM}}$ and $\mathrm{KSFM}^{\mathrm{TM}}$ media contain bovine pituitary extraction supplement (BPE), which is a crude animal product of undefined composition that can be a potential source of contaminants. In Epilife $^{\mathrm{TM}}$ serum-free medium, the BPE is replaced by purified serum albumin, transferrin, recombinant human Insulin-like Growth Factor (rhIGF-1), and prostaglandin E2 (PGE-2), thus maintaining a highly purified animal-free product system. BPE has also been replaced in fabrication of our human EVPOMEs to gain FDA approval in anticipation of our human clinical trials. ${ }^{5}$

The culture kinetics of the canine oral keratinocytes in our study showed that cell proliferation decreased markedly after passage three resulting in a shorter lifespan of canine cells when compared with human oral keratinocytes grown in the same medium. This would seem to imply that different growth conditions and/or media are necessary to optimize the culturing of canine oral keratinocytes. The amplification of the canine oral keratinocytes within the first three passages was sufficient to allow us to harvest ample cells for fabrication of the necessary equivalents for our in vitro and planned in vivo studies. The average cell size increased from passage one to passage five in association with an increase in intracytoplasmic particles, which correlated with the decrease in cell replication and increase in cell senescence. In contrast to passage one to passage four cells, the forward scatter of passage five cells showed two-peak patterns. In this study, smaller cells appeared among larger cell colonies in passage five and passage six culture cells. The second growth surge, observed in later passages in extended culture periods, has previously been observed by Lauer et al. $^{20}$ It appears that cells of a smaller size may have a critical role in the proliferative potential of keratinocytes seen in later culture periods as this is consistent with the physical stature of stem cells. $^{21}$

Investigations have shown that keratinocytes grown in serum-free medium behave differently than keratinocytes grown in medium containing serum. ${ }^{22} \mathrm{Sev}-$ eral studies have suggested that the serum-free medium encourages keratinocytes to change to a proliferative phenotype. ${ }^{15,23}$ Unfortunately, the serum-containing medium used in these studies was not depleted of calcium, and the actual concentration present was not known. This makes it difficult to determine the effects of the calcium concentration versus the presence or absence of serum to explain the difference in proliferation and differentiation effects on keratinocyte in these varied media. Our data showed that Epilife ${ }^{\mathrm{TM}}$ serum-free medium supported rapid keratinocytes amplification at the earlier stages in our culture system, but was not as long-lived as chelexed serum-contained medium, devoid of calcium, in the later phases (data not shown). The study by Altankov et al. ${ }^{22}$ revealed that the human keratinocytes cultured in a serum-free medium had an adherent phenotype based on integrin $\beta 1$ expression but when human keratinocytes were cultured in a serumcontained medium the cells changed to a migratory phenotype. In addition, keratinocytes that grew well in a serum-free medium failed to reorganize fibronectin as a matrix substrate which binds to integrin $\beta 1 .^{22}$ A reversible phenotypic alteration of keratinocytes in vitro, such as adherent, proliferative, or motile, may depend on adherent inhibition factors contained in serum. According to Marcelo et al., ${ }^{24}$ the normalization of the fatty acids composition of the cell membrane gave rise to a slower growth rate, a decreased passage potential, and a more differentiated cell morphology in serum-free condition. Because a $10-20 \%$ serum-supplemented medium does not affect cultured keratinocytes to normalize fatty acid composition of cell membrane, essential fatty acids deficiency in a serum-free culture system appears to outperform a serum-contained medium to expand a canine keratinocyte population rapidly and to be able to obtain a 
sufficient number of keratinocytes in a shorter period of time.

Oral mucosa is classified into two basic types (excluding the specialized mucosa of the tongue) according to the pattern of keratinization: keratinized or masticatory mucosa-which includes the palate and attached mucosa and nonkeratinized mucosa which includes the buccal and floor of the mouth mucosa. ${ }^{25}$ In our investigation, we choose to use keratinocytes from buccal mucosa for several reasons: first, it is plentiful because both cheeks and vestibules can be a source of tissue; secondly, this donor site was relatively easy to close because of the laxity of mucosa in this area; and finally, the palate is to be the recipient site for our in vivo grafting of the canine EVPOME and thus needed to remain unscathed. Our histological results demonstrated that the keratinization pattern of the canine equivalent was consistent with the original oral buccal mucosa only lacking in the deep ridges and pigmentation seen in native canine oral mucosa. The immunohistochemical staining showed that the day 18 equivalent had a similar p63, Ki-67, and involucrin expression pattern, as native canine buccal mucosa that substantiate the efficacy of the use of the canine EVPOME in our studies.

p63 transcription factor, a p53 homolog, has been identified as an essential factor for regenerative proliferation in epithelial development. p63 ${ }^{-/-}$mice have major defects in their limb and craniofacial development, as well as a striking absence of stratified epithelia. ${ }^{26}$ Pellegrini et al. found that p63 is abundantly expressed by epidermal and limbal holoclones, but is undetectable in paraclones. ${ }^{27}$ Transit amplifying keratinocytes have a reduction in p63 expression immediately after they leave the stem cell compartment implying p63 may be a keratinocyte stem cell marker. ${ }^{27}$ Immunohistochemical stain of p63 in human epithelial tissues shows that p63 staining is localized to the basal cells of various epithelia. ${ }^{26}$ In our experiment, p63-positive nuclei were found in the basal cells in native canine oral buccal mucosa, especially between the dermal ridges. The same staining pattern was shown in our canine EVPOMEs. Ki-67, a cell proliferation-associated human nuclear antigen, is expressed throughout the cell cycle, reaching maximum at G2/M. Ki-67 expression is of value in detecting dividing keratinocytes in the human epidermis. ${ }^{28}$ Although the Ki-67 expression in native canine oral buccal mucosa, as well as equivalents, was mainly found in the basal cells layers, the percent positive cells was much lower than seen with p63. This implies that most p63-positive cells are in a quiescent phase (G0) of cell cycle instead of a proliferating phase (S/G2/ $\mathrm{M})$, which is a known characteristic of stem cells. ${ }^{29,30}$

Investigators have performed human clinical trials without being able to definitively determine if the keratinocytes present at the grafted site are trans- planted or resident keratinocytes migrating in from the periphery of the wound. ${ }^{2,31}$ This is a critical point to assess because if the transplanted keratinocytes on the EVPOME do not survive the grafting procedure, it calls into question the efficacy of using them as a source of gene products once they are transfected. Therefore, in this investigation, we attempted to label keratinocytes, on the soon-to-be-grafted canine EVPOME, by transducing them with a reporter gene, GFP, using a lentiviral vector, in order to track the oral keratinocytes on grafted EVPOMEs. Lentivirus vectors are a family of retroviral vectors, that have several advantages in cellular transduction: they complement the features of efficient integration in the chromatin, there is an absence of viral genes from the genetic information transferred to the recipient, there is a lack of preexisting immunity in the recipient, and, even more important, they are able to infect nondividing cells. ${ }^{32}$ By injecting a lentiviral vector into human skin tissue, it will successfully target all major cell types within the human skin including keratinocytes. In addition, studies using lentiviral vectors encoding for human erythropoietin, transfected into transplanted cells, were able to produce a dose-dependent increase in serum human erythropoietin level. ${ }^{33}$ This is important because we have future studies planned to transfect our oral keratinocytes with a functional gene in place of GFP. Although the lentiviral vector was derived from human immunodeficiency virus-1, it has become an invaluable gene transfer tool by showing its efficacy and biosafety by changing it to a nonprimate immunodeficiency virus vector. ${ }^{32}$ The feline immunodeficiency virus vector (FIV) we used in our experiment showed a maximum of $30 \%$ transduction efficiency and a long-term expression of GFP within our canine equivalent. Also of importance, the transducted oral keratinocytes still possessed the ability to form stratified cell layers once seeded on the AlloDerm $^{\circledR}$, which suggested that their normal function was not influenced by the incorporated viral gene. By using GFP-transducted EVPOME, in vivo, the source of keratinocytes that covers the wound, at last, can be assessed. In addition, the feature of infecting nondividing cells by the lentiviral vector is an advantage over other retroviral vectors because one can now target the slowly dividing stem cell. ${ }^{34-36}$

In conclusion, canine EVPOMEs were successfully fabricated in an identical manner to our human EVPOMEs. The canine EVPOMEs were similar in nature to native canine oral buccal mucosa. GFP-transfected canine oral keratinocytes used to fabricate canine EVPOMEs could be identified within the canine EVPOME. In vivo studies utilizing the GFP-transfected canine EVPOMEs are presently underway.

The authors thank the Vector Core in University of Michigan for technical assistance on the transfection studies. They 
gratefully acknowledge Drs. Blake J. Roessler and Cynthia L. Marcelo for their valuable suggestions and Dr. Takayoshi Tobita and Judith Schmitt for technical support and guidance of the tissue culture studies. This work was supported by grants from the Kato Memorial Bioscience Foundation (K. Izumi) and US PHS DE 13417 (S. E. Feinberg).

\section{References}

1. Feinberg SE. Tissue-engineered mucosa graft for reconstruction of the intraoral lining after freeing of the tongue: a clinical and immunohistologic study. Oral Maxillofac Surg 2001;59: 175-177. Discussion.

2. Izumi K, Feinberg SE, Iida A, Yoshizawa M. Intraoral grafting of an ex vivo produced oral mucosa equivalent: a preliminary report. Int J Oral Maxillofac Surg 2003;32:188-197.

3. Izumi K, Terashi H, Marcelo CL, Feinberg SE. Development and characterization of a tissue-engineered human oral $\mathrm{mu}-$ cosa equivalent produced in a serum-free culture system. J Dent Res 2000;79:798-805.

4. Izumi K, Feinberg SE, Terashi H, Marcelo CL. Evaluation of transplanted tissue-engineered oral mucosa equivalents in severe combined immunodeficient mice. Tissue Eng 2003;9:163174.

5. Izumi K, Song J, Feinberg SE. Development of a tissue engineered human oral mucosa: from the bench to the bed side. Cells Tissues Organs 2004;176:134-152.

6. Chan LS. Mucous membrane pemphigoid. Dermatol Clin 2001; 19:703-711.

7. Nicholls PK, Doorbar J, Moore RA, Peh W, Anderson DM, Stanley MA. Detection of viral DNA and E4 protein in basal keratinocytes of experimental canine oral papillomavirus lesions. Virology 2001;284:82-98.

8. Dillberger JE, Altman NH. Focal mucinosis in dogs: seven cases and review of cutaneous mucinoses of man and animals. Vet Pathol 1986;23(2):132-139.

9. Crameri FM, Varvayanis M, Cromie BR, Rekers WL, Suter MM. Serum-free conditions for the long term growth and differentiation of neoplastic canine keratinocytes. Exp Dermatol 1997;6:147-155.

10. Wilkinson JE, Smith C, Suter M, Lewis RM. Long-term cultivation of canine keratinocytes. J Invest Dermatol 1987;88:202206.

11. Ophof R, van Rheden RE, Von den HJ, Schalkwijk J, KuijpersJagtman AM. Oral keratinocytes cultured on dermal matrices form a mucosa-like tissue. Biomaterials 2002;23:3741-3748.

12. Suter MM, Pantano DM, Flanders JA, Augustin-Voss HG, Dougherty EP, Varvayanis M. Comparison of growth and differentiation of normal and neoplastic canine keratinocyte cultures. Vet Pathol 1991;28:131-138.

13. Feinberg SE, Krishnan V, Gordillo G, Shuler CF. Intraoral grafting of a canine full-thickness oral mucosal equivalent produced in vitro. J Oral Maxillofac Surg 1989;47:712-718.

14. Ian Freshney R. Culture of animal cells. New York: John Wiley \& Sons; 2000. p 321-323.

15. Izumi K, Takacs G, Terashi H, Feinberg SE. Ex vivo development of a composite human oral mucosal equivalent. J Oral Maxillofac Surg 1999;57:571-577.

16. van der Valk J, Mellor D, Brands R, Fischer R, Gruber F, Gstraunthaler G, Hellebrekers L, Hyllner J, Jonker FH, Prieto P, Thalen M, Baumans V. The humane collection of fetal bovine serum and possibilities for serum-free cell and tissue culture. Toxicol In Vitro 2004;18:1-12.

17. Selvaggi TA, Walker RE, Fleisher TA. Development of antibodies to fetal calf serum with arthus-like reactions in human immunodeficiency virus-infected patients given syngeneic lymphocyte infusions. Blood 1997;89:776-779.

18. Marchese C, Felici A, Visco V, Lucania G, Igarashi M, Picardo M, Frati L, Torrisi MR. Fibroblast growth factor 10 induces proliferation and differentiation of human primary cultured keratinocytes. J Invest Dermatol 2001;116:623-628.

19. Bikle DD, Ng D, Tu CL, Oda Y, Xie Z. Calcium- and vitamin D-regulated keratinocyte differentiation. Mol Cell Endocrinol 2001;177:161-171.

20. Lauer G, Siegmund C, Hubner U. Influence of donor age and culture conditions on tissue engineering of mucosa autografts. Int J Oral Maxillofac Surg 2003;32:305-312.

21. Dazard J-E, Piette J, Basset-Seguin N, Blanchard J-M, Gandarillas A. Switch from p53 to MDM2 as differentiating human keratinocytes lose their proliferative potential and increase in cellular size. Oncogene 2000;19:3693-3705.

22. Altankov G, Hecht J, Dimoudis N. Serum-free cultured keratinocytes fail to organize fibronectin matrix and possess different distribution of beta-1 integrins. Exp Dermatol 2001;10:8089.

23. Kaighn ME, Carnalier RF, Bertolero F, Saffiotti U. Spontaneous establishment and characterization of mouse keratinocyte cell lines in serum-free medium. In Vitro Cell Dev Biol 1988;24: 845-854.

24. Marcelo CL, Rhodes LM, Dunham WR. Normalization of essential-fatty-acid-deficient keratinocytes requires palmitic acid. J Invest Dermatol 1994;103:564-568.

25. Strachan DS. Histology of the oral mucosa and tonsils. In: Avery JK, editor. Oral development and histology. New York: Thieme Medical Publishers; 1994. p 298-320.

26. Yang A, Kaghad M, Wang Y, Gillett E, Fleming MD, Dotsch V, Andrews NC, Caput D, McKeon F. p63, a p53 homolog at 3q27-29, encodes multiple products with transactivating, death-inducing, and dominant-negative activities. Mol Cell 1998;2:305-316.

27. Pellegrini G, Dellambra E, Golisano O, Martinelli E, Fantozzi I, Bondanza S, Ponzin D, McKeon F, De Luca M. p63 identifies keratinocyte stem cells. Proc Natl Acad Sci USA 2001;98(6): 3156-3161.

28. Andreadis ST, Hamoen KE, Yarmush ML, Morgan JR. Keratinocyte growth factor induces hyperproliferation and delays differentiation in a skin equivalent model system. FASEB J 2001;15:898-906.

29. Watt FM. Stem cell fate and patterning in mammalian epidermis. Curr Opin Genet Dev 2001;11:410-417.

30. Janes SM, Lowell S, Hutter C. Epidermal stem cells. J Pathol 2002;197:479-491.

31. Lauer G, Schimming R. Tissue-engineered mucosa graft for reconstruction of the intraoral lining after freeing of the tongue: a clinical and immunohistologic study. J Oral Maxillofac Surg 2001;59:169-175.

32. Vigna E, Naldini L. Lentiviral vectors: excellent tools for experimental gene transfer and promising candidates for gene therapy. J Gene Med 2000;2(5):308-316.

33. Baek SC, Lin Q, Robbins PB, Fan H, Khavari PA. Sustainable systemic delivery via a single injection of lentivirus into human skin tissue. Hum Gene Ther 2001;12:1551-1558.

34. Kuhn U, Terunuma A, Pfutzner W, Foster RA, Vogel JC. In vivo assessment of gene delivery to keratinocytes by lentiviral vectors. J Virol 2002;76:1496-1504.

35. Chen M, Li W, Fan J, Kasahara N, Woodley D. An efficient gene transduction system for studying gene function in primary human dermal fibroblasts and epidermal keratinocytes. Clin Exp Dermatol 2003;28:193-199.

36. Bickenbach JR, Dunnwald M. Epidermal stem cells: characteristics and use in tissue engineering and gene therapy. Adv Dermatol 2000;16:159-183. 